\title{
Photon-Pair Generation using Coupled Silicon Microring Resonators (1201308-PO)
}

\section{SHAYAN MOOKHERJEA}

Department of Electrical and Computer Engineering, University of California, San Diego, MC 0407 La Jolla CA 92093-0407 USA

Email:smookher@ucsd.edu

Summary of a Project Outcomes report of research funded by the U.S. National Science Foundation under Project Number 1201308. The physical principles of photon-Pair generation in coupled silicon microring resonators were studied theoretically and experimentally.

\section{Introduction}

Microring resonators can be fabricated in silicon photonics with low loss (high quality factor). Such devices may be useful for on-chip nonlinear optics such as wavelength conversion through four-wave mixing (FWM) and photon-pair generation through spontaneous four-wave mixing (SFWM). Figure 1 shows the difference between single resonators and coupled resonators, using the CROW concept (coupled resonator optical waveguides). Research in this project seeks to understand how the physics of optical propagation in CROWs impacts the photon pair generation properties.

a)
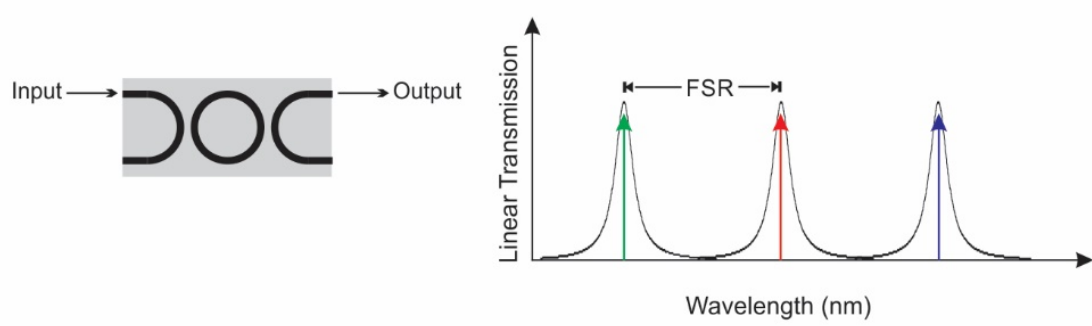

b)
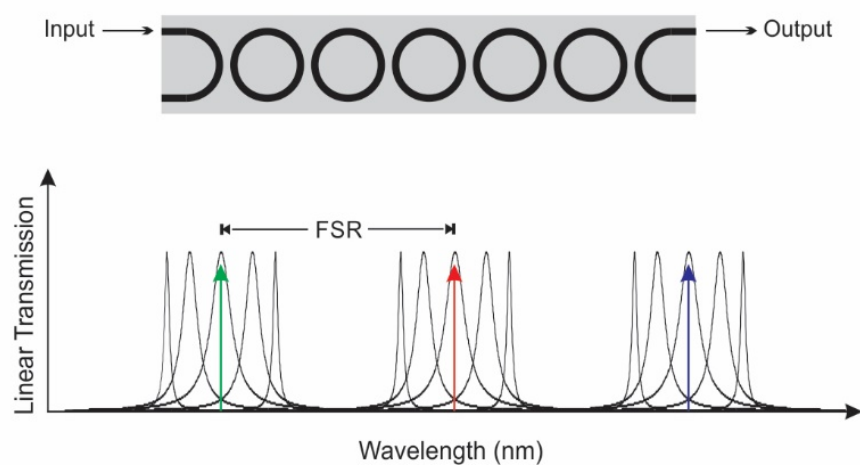

Figure 1 (a) Single ring resonator and (b) a Coupled-Resonator Optical Waveguide (CROW). The transmission spectrum are sketched. Upward pointing arrows denote potential locations for the signal, pump and idler wavelengths. 
In both cases, the light in the resonator is resonantly enhanced, i.e., the local intensity in the waveguide cross-section is higher than in a conventional waveguide. This leads to a higher efficiency of nonlinear interactions. However, a CROW has multiple peaks within a transmission passband whereas a single resonator has one resonance (per free spectral range). The number of peaks in a CROW passband equal to the number of resonators. When measured with a swept-wavelength laser, the transmission may not resolve the individual peaks, depending on their linewidth. When a continuous wave laser is positioned at a particular supermode frequency, the resulting transmission is quasi-Lorentzian, which is indicated by the schematic in Fig. 1(b). A supermode describes a collective resonance of all $\mathrm{N}$ resonators (see Figure 2), and so the light-matter interaction length is longer than in a single resonator.

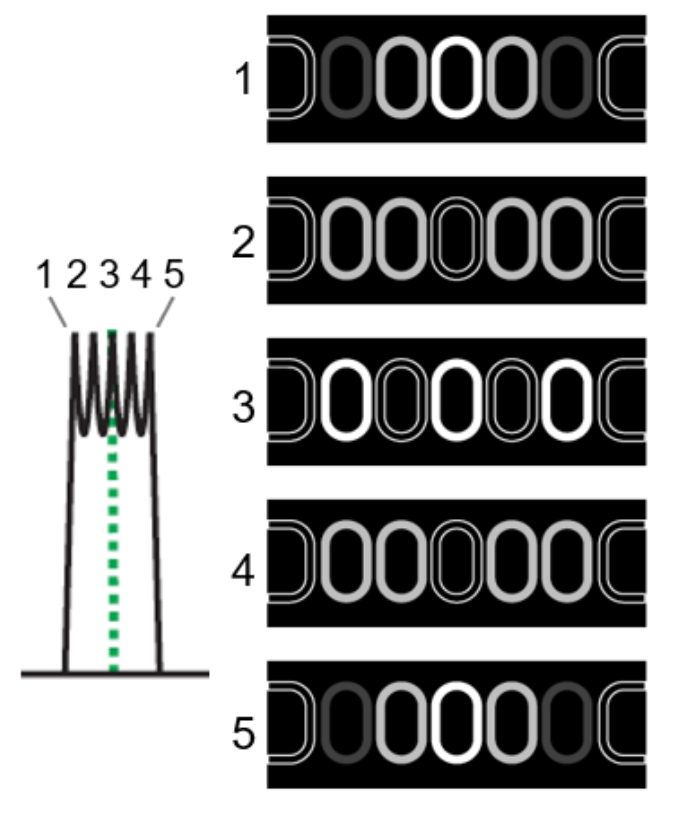

Figure 2 Cartoon representation of supermodes in a CROW. Each of the transmission resonance peaks corresponds to an eigenvalue, and the corresponding eigenmode (called a supermode) is a collective resonance of all the resonators with different amplitude coefficients.

Light propagates in a CROW by nearest-neighbor coupling. A tight-binding model was used in early studies of optical CROWs [1], [2] and was reformulated in a matrix eigenvalue problem to study the modes and resonance frequencies of the coupled-resonator system [3]. A more detailed and accurate computational method was developed for studying CROWs [4], [5] which are based on numerical insights into coupled-mode theory which we developed in a previous project [6]. A potential application of CROW filters is as part of an integrated photonic circuit following a nonlinear mixer device [7]. CROWs can also be used as tunable dispersion compensation devices [8] or as a laser [9]. Like single microring resonators, CROWs can be used for wavelength conversion through four-wave mixing [10], and for the closely-related process 
of generating entangled photon-pairs at room temperature using optical pumping at moderately low pump powers (milliwatts or less) [11], [12].

\section{Joint Spectral Intensity}

A CROW consisting of an array of coupled microring resonators was fabricated using CMOS-compatible processes on SOI wafers with $220 \mathrm{~nm}$ silicon layer height. The device was pumped using $3 \mathrm{~mW}$ average power in waveguide ( $8 \mathrm{~ns}$ pulse width, $15 \mathrm{MHz}$ repetition rate) of TE-polarized light $\left(\lambda_{\mathrm{P}} \approx 1556 \mathrm{~nm}\right)$. The spontaneous four-wave mixing nonlinear optical process generates two TEpolarized, frequency-non-degenerate entangled photons at wavelengths conventionally called "signal" $\left(\lambda_{\mathrm{s}} \approx 1541 \mathrm{~nm}\right)$ and "idler" $\left(\lambda_{\mathrm{I}} \approx 1571 \mathrm{~nm}\right)$. The dispersion of the microring CROW waveguide has been studied [13].

The JSI is an important quantity in characterizing a pair source, since it is the squared-magnitude of the wavelength-resolved amplitude coefficient of the two-photon state. States with different entanglement properties are expected to have different JSIs. In a CROW, the JSI can be calculated by measuring the transmission spectrum at the signal and idler passbands, and then forming the two-dimensional "grid" of frequencies as shown in Fig. 3.

The pump selects a diagonal slice along the 2D grid. The resulting JSI can be changed from a single-peak shape to multiple peaks by tuning the wavelength of the resonators or the pump. The single-peak JSI is characterized by a low Schmidt number of approximately 1.9 , resulting from an elliptical shape of the JSI. The multi-peak JSI has a higher Schmidt number (about 6).
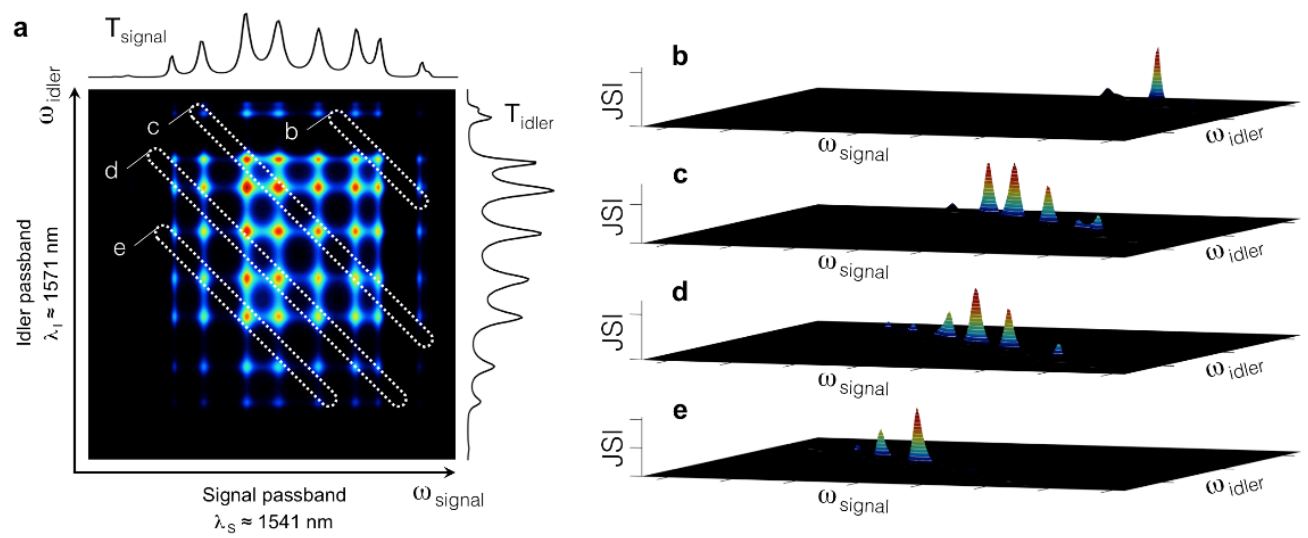

Figure 3 Surface plot of the coefficient in the two-photon wavefunction vs. the optical frequencies in the "signal" and "idler" spectral ranges. Calculated Joint Spectral Intensities (JSI) are shown in panels b-e for 4 distinct choices of the pump wavelength (or equivalently, chip temperature). The JSIs are normalized to unity in each case. 
(a)

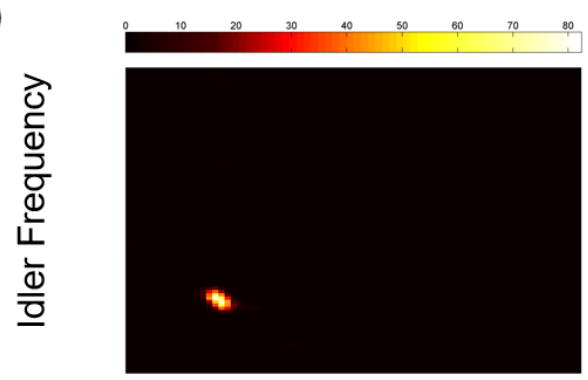

Signal Frequency

(c)

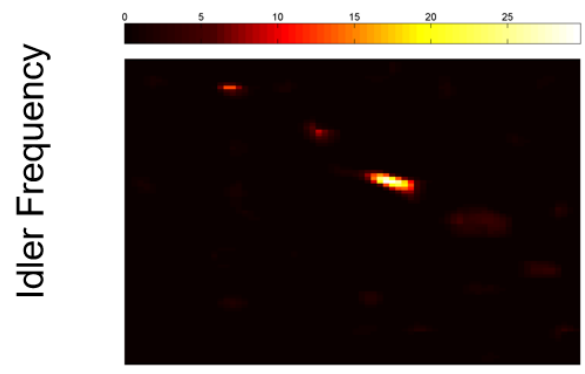

Signal Frequency (b)

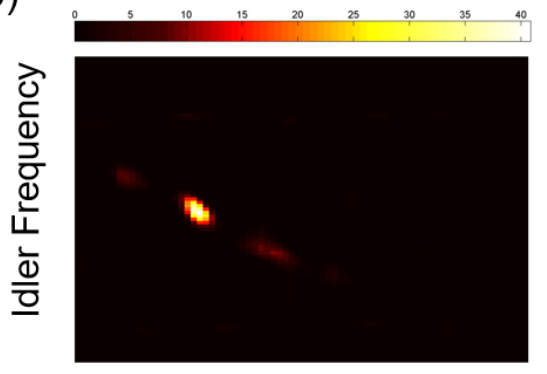

Signal Frequency

(d)

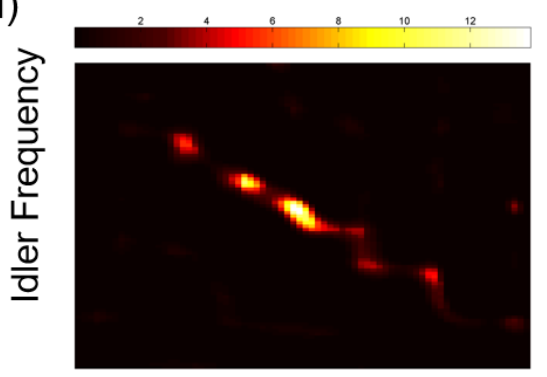

Signal Frequency

Figure 4 Experimental measurements of the two-photon spectrum (joint spectral intensity) near the signal wavelength of $1541 \mathrm{~nm}$ and the idler wavelength of $1571 \mathrm{~nm}$ (over an extent of about $100 \mathrm{GHz} \times 100 \mathrm{GHz})$

Figure 4 shows experimentally measured JSI after deconvolution to reduce the artifacts from measurement using optical filters that have a relatively broad spectral roll-off. By tuning the wavelength of the pump, different JSI's were generated, which correspond to quantum two-photon states with different entanglement properties [14]. This is not possible when generating photon-pairs using a single microring resonator.

\section{Additional Experimental Considerations.}

Off resonance

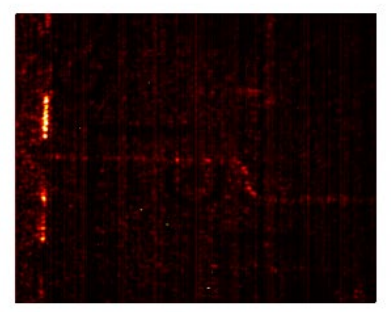

Near resonance

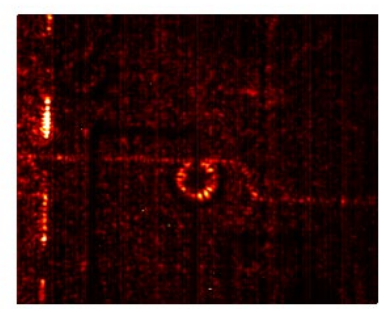

On resonance

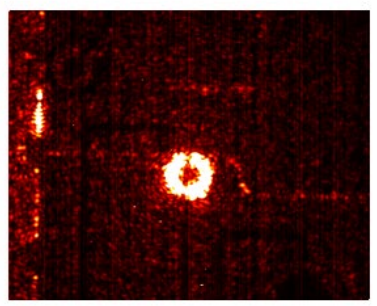

Figure 5 Infrared camera images (through a microscope) of light near $1550 \mathrm{~nm}$ guided in a bus waveguide near a microring resonator. The camera collects scattered light. The ring is much brighter than the waveguide when on resonance. 
Infrared imaging is a useful experimental technique to study both single ring resonators and CROW structures [15], [16]. We observe the silicon microring resonator (or CROW) as the pump wavelength or the chip temperature is varied (see Fig. 5) and a substantial increase in brightness of the captured image is seen when the pump is near resonance. Then, we perform fine tuning of the wavelength or temperature in order to maximize the counts.

The $\mathrm{p}-\mathrm{i}-\mathrm{n}$ diode in the waveguide cross-section plays an important role in these microring resonators [17]. Under reverse bias, a photocurrent can be measured when the optical pump is resonant with the microring as shown in Figure 6. Typically, the measured photocurrent is in the microampere range, so that the pump is not depleted. Note that this diagnostic technique does not require light to be measured at an output port, and also has a low noise floor, since only the light that is in the resonator is measured.

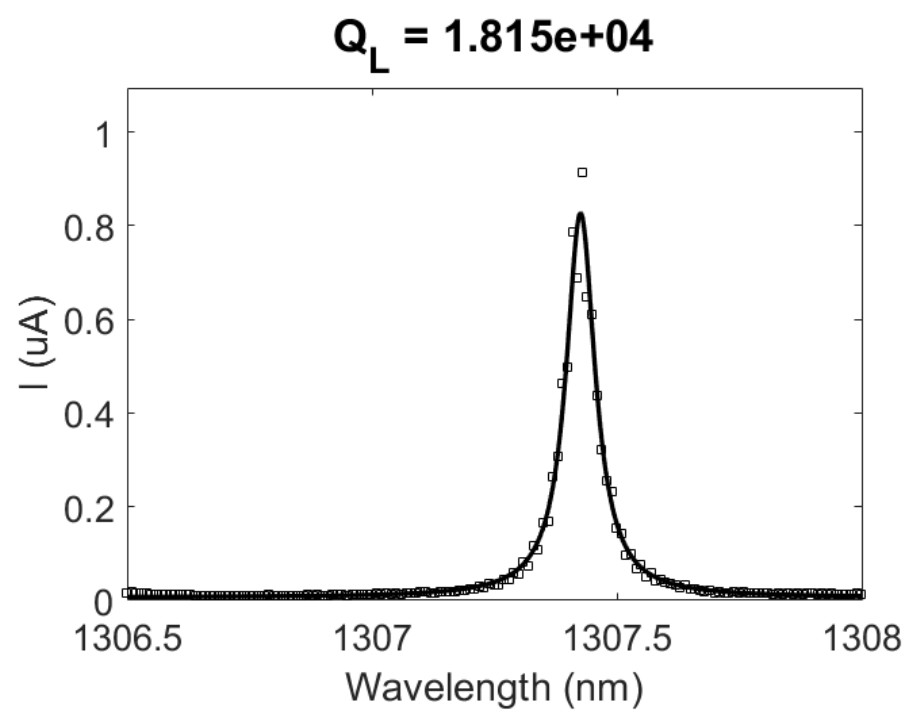

Figure 6 Reverse biased photocurrent measured for a silicon microring resonator near $1310 \mathrm{~nm}$. From the measurement, the loaded quality factor (QL) can be calculated, just as if the transmission was measured using a fiber aligned to an output port of the chip.

Figure 7 shows a screen capture of a software program written to monitor the photocurrent and see the image captured by the infrared camera. These two methods are used in experiments to continuously verify that the pump is aligned to a resonance.

High-Q silicon microring resonators are sensitive to fabrication disorder. For precisely trimming resonators we have studied field-induced local oxidation of $\mathrm{Si}$ to $\mathrm{SiO}_{2}$ via a chemical reaction near an electrically-biased conducting atomic-force microscope tip [18], [19]. There are other trimming and tuning methods that are under development. The same thermal tuning mechanism used for optical switching[20] can often be used during operation to bias and 
stabilize the device at a particular wavelength, although the bias point is obviously lost when the electrical power is disconnected.

Another potential difficulty in using certain types of single racetrack resonators for achieving a high pair generation rate (PGR) is that a long coupling segment itself can have a significant dispersive [21] or nonlinear effect [22] compared to the phase shift of the ring itself. These effects are partially mitigated in the CROW structure by achieving the same PGR at a lower power level.

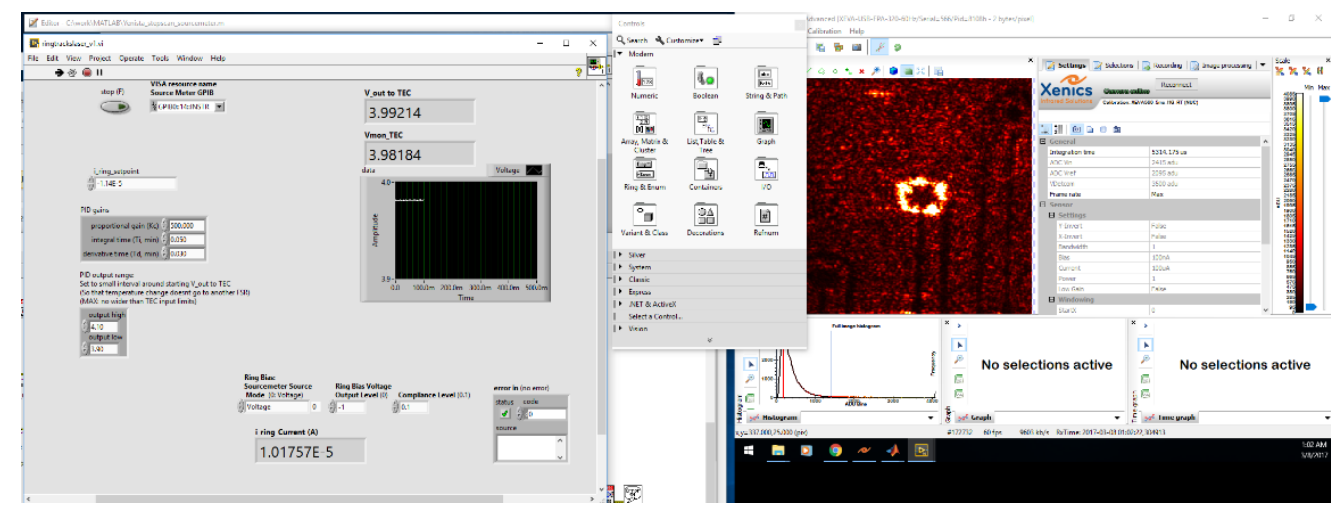

Figure 7 (left panel) Monitoring of photocurrent using a software program that reads out the current through a sourcemeter. (right panel) Monitoring the infrared image of the ring through a software interface provided by the camera manufacturer.

Longer CROW devices consisting of passive silicon resonators have been realized [23]. CROWs with $p-n$ junctions across the waveguide cross-section have also been shown [24]. An alternative fast but low-loss tuning mechanism would be achieved by incorporating a ferro-electric material such as lithium niobate, as has been shown with conventional Mach-Zehnder interferometers in a hybrid silicon platform, but not yet with CROWs [25].

The optical Kerr effect that is closely related to four-wave mixing and photon pair generation can also result in a rich variety of other phenomena, such as soliton formation [26] and pattern formation [27] and pulse storage (optical memory)[28], [29]. Most of these subjects have been theoretically investigated but not yet experimentally shown, but with increasing skill in fabrication and control over biasing and stabilization as shown above, we are optimistic of making progress in these areas as well.

\section{Open-Access Reporting Initiative}

PRAISE: This open-access document is provided in support of our PRAISE

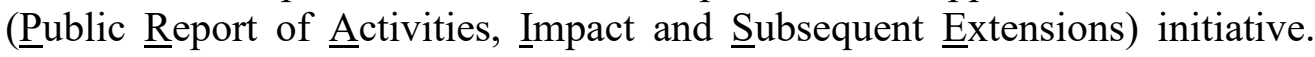
What is it? An open-access document shared with the public which describes 
the research outcomes of publicly-funded projects such as those funded by the U.S. NSF (National Science Foundation).

\section{References}

[1] S. Mookherjea, D. S. Cohen, and A. Yariv, "Nonlinear dispersion in a coupled-resonator optical waveguide," Opt. Lett., vol. 27, no. 11, p. 933, Jun. 2002, doi: 10.1364/OL.27.000933.

[2] S. Mookherjea and A. Yariv, "Optical pulse propagation in the tightbinding approximation," Opt. Express, vol. 9, no. 2, p. 91, Jul. 2001, doi: 10.1364/OE.9.000091.

[3] S. Mookherjea, "Spectral characteristics of coupled resonators," J. Opt. Soc. Am. B, vol. 23, no. 6, p. 1137, Jun. 2006, doi: 10.1364/JOSAB.23.001137.

[4] S. Mookherjea and M. A. Schneider, "Avoiding bandwidth collapse in long chains of coupled optical microresonators," Opt. Lett., vol. 36, no. 23, p. 4557, Dec. 2011, doi: 10.1364/OL.36.004557.

[5] M. L. Cooper and S. Mookherjea, "Modeling of Multiband Transmission in Long Silicon Coupled-Resonator Optical Waveguides," IEEE Photon. Technol. Lett., vol. 23, no. 13, pp. 872-874, Jul. 2011, doi: 10.1109/LPT.2011.2141657.

[6] M. L. Cooper and S. Mookherjea, "Numerically-assisted coupled-mode theory for silicon waveguide couplers and arrayed waveguides," Opt. Express, vol. 17, no. 3, p. 1583, Feb. 2009, doi: 10.1364/OE.17.001583.

[7] J. R. Ong, R. Kumar, and S. Mookherjea, "Silicon microring-based wavelength converter with integrated pump and signal suppression," Opt. Lett., vol. 39, no. 15, p. 4439, Aug. 2014, doi: 10.1364/OL.39.004439.

[8] S. Mookherjea, "Using gain to tune the dispersion relation of coupledresonator optical waveguides," IEEE Photon. Technol. Lett., vol. 18, no. 5, pp. 715-717, Mar. 2006, doi: 10.1109/LPT.2006.871144.

[9] S. Mookherjea, "Semiconductor coupled-resonator optical waveguide laser," Appl. Phys. Lett., vol. 84, no. 17, pp. 3265-3267, Apr. 2004, doi: 10.1063/1.1719278.

[10] J. R. Ong et al., "Low-power continuous-wave four-wave mixing in silicon coupled-resonator optical waveguides," Opt. Lett., vol. 36, no. 15, pp. 2964-2966, 2011.

[11] J. R. Ong and S. Mookherjea, "Quantum light generation on a silicon chip using waveguides and resonators," Opt. Express, vol. 21, no. 4, p. 5171, Feb. 2013, doi: 10.1364/OE.21.005171.

[12] R. Kumar, M. Savanier, J. R. Ong, and S. Mookherjea, "Entanglement measurement of a coupled silicon microring photon pair source," Opt. Express, vol. 23, no. 15, p. 19318, Jul. 2015, doi: 10.1364/OE.23.019318. 
[13] M. L. Cooper et al., "Waveguide dispersion effects in silicon-oninsulator coupled-resonator optical waveguides," Opt. Lett., vol. 35, no. 18, p. 3030, Sep. 2010, doi: 10.1364/OL.35.003030.

[14] R. Kumar, J. R. Ong, M. Savanier, and S. Mookherjea, "Controlling the spectrum of photons generated on a silicon nanophotonic chip," Nat Commun, vol. 5, no. 1, p. 5489, Dec. 2014, doi: 10.1038/ncomms6489.

[15] M. L. Cooper, G. Gupta, J. S. Park, M. A. Schneider, I. B. Divliansky, and S. Mookherjea, "Quantitative infrared imaging of silicon-on-insulator microring resonators," Opt. Lett., vol. 35, no. 5, p. 784, Mar. 2010, doi: 10.1364/OL.35.000784.

[16] S. Mookherjea and H. R. Grant, "High dynamic range microscope infrared imaging of silicon nanophotonic devices," Opt. Lett., vol. 37, no. 22, p. 4705, Nov. 2012, doi: 10.1364/OL.37.004705.

[17] M. Savanier, R. Kumar, and S. Mookherjea, "Optimizing photon-pair generation electronically using a $p-i-n$ diode incorporated in a silicon microring resonator," Appl. Phys. Lett., vol. 107, no. 13, p. 131101, Sep. 2015, doi: 10.1063/1.4932047.

[18] Y. Shen, I. B. Divliansky, D. N. Basov, and S. Mookherjea, "Perfect set-and-forget alignment of silicon photonic resonators and interferometers," in Optical Fiber Communication Conference/National Fiber Optic Engineers Conference 2011, Los Angeles, California, 2011, p. PDPC3. doi: 10.1364/OFC.2011.PDPC3.

[19] Y. Shen, I. B. Divliansky, D. N. Basov, and S. Mookherjea, "Electricfield-driven nano-oxidation trimming of silicon microrings and interferometers," Opt. Lett., vol. 36, no. 14, p. 2668, Jul. 2011, doi: 10.1364/OL.36.002668.

[20] R. Aguinaldo et al., "Wideband silicon-photonic thermo-optic switch in a wavelength-division multiplexed ring network," Opt. Express, vol. 22, no. 7, p. 8205, Apr. 2014, doi: 10.1364/OE.22.008205.

[21] R. Aguinaldo, Yiran Shen, and S. Mookherjea, "Large Dispersion of Silicon Directional Couplers Obtained via Wideband Microring Parametric Characterization," IEEE Photon. Technol. Lett., vol. 24, no. 14, pp. 1242-1244, Jul. 2012, doi: 10.1109/LPT.2012.2198639.

[22] S. Mookherjea and M. A. Schneider, "The nonlinear microring adddrop filter," Opt. Express, vol. 16, no. 19, p. 15130, Sep. 2008, doi: 10.1364/OE.16.015130.

[23] M. L. Cooper et al., "235-ring Coupled-Resonator Optical Waveguides," in Conference on Lasers and Electro-Optics 2010, San Jose, California, 2010, p. CTuHH3. doi: 10.1364/CLEO.2010.CTuHH3.

[24] S. Mookherjea, J. R. Ong, X. Luo, and L. Guo-Qiang, "Electronic control of optical Anderson localization modes," Nature Nanotech, vol. 9, no. 5, pp. 365-371, May 2014, doi: 10.1038/nnano.2014.53. 
[25] X. Wang, P. O. Weigel, J. Zhao, M. Ruesing, and S. Mookherjea, "Achieving beyond-100-GHz large-signal modulation bandwidth in hybrid silicon photonics Mach Zehnder modulators using thin film lithium niobate," APL Photonics, vol. 4, no. 9, p. 096101, Sep. 2019, doi: 10.1063/1.5115243.

[26] S. Mookherjea and A. Yariv, "Kerr-stabilized super-resonant modes in coupled-resonator optical waveguides," Phys. Rev. E, vol. 66, no. 4, p. 046610, Oct. 2002, doi: 10.1103/PhysRevE.66.046610.

[27] B. Crosignani, A. Yariv, and S. Mookherjea, "Nonparaxial spatial solitons and propagation-invariant pattern solutions in optical Kerr media," Opt. Lett., vol. 29, no. 11, p. 1254, Jun. 2004, doi: 10.1364/OL.29.001254.

[28] S. Mookherjea and A. Yariv, "Optical pulse propagation and holographic storage in a coupled-resonator optical waveguide," Phys. Rev. $E$, vol. 64, no. 6, p. 066602 , Nov. 2001, doi: 10.1103/PhysRevE.64.066602.

[29] S. Mookherjea and A. Yariv, "Pulse propagation in a coupled resonator optical waveguide to all orders of dispersion," Phys. Rev. E, vol. 65, no. 5, p. 056601, Apr. 2002, doi: 10.1103/PhysRevE.65.056601. 